\title{
Association between PNPLA8 gene polymorphism and schizophrenia in male patients
}

\author{
Q Yu, Phd; Y Yu, Phd; J Shi, BSc; H Sang, MD \\ ${ }^{1}$ Department of Epidemiology and Biostatistics, Center of Medical Genomics, School of Public Health, Jilin University, Changchun 130021, China \\ ${ }^{2}$ Psychiatric Hospital of Changchun City, Changchun 130052, China
}

Corresponding author: Q Yu (yujoan_2001@163.com)

\begin{abstract}
Abnormal phospholipid metabolism in the brain plays an important role in neuropsychiatric diseases. Phospholipase A2 is crucial for maintaining normal neuro-physiological function. The aim of this study was to investigate the association between polymorphisms of the membrane-associated calcium-independent phospholipase A2 gamma (PNPLA8) gene and schizophrenia in Han Chinese in north China. The PCR-based ligase detection reaction was applied to detect 3 single nucleotide polymorphisms (SNPs) in the PNPLA8 gene among 201 Chinese pedigrees. The genotypic frequency of the PNPLA8 polymorphisms did not deviate from the Hardy-Weinberg equilibrium both in affected offspring and parental groups. Haploid relative risk (HRR) and transmission disequilibrium tests (TDT) showed that the 3 SNPs were not associated with schizophrenia ( $p>0.05)$, but further analysis with TDT showed that the rs40876 polymorphism was associated with schizophrenia in males $\left(\chi^{2}=4.667, p=0.031\right)$. Our data suggest that rs40876 in PNPLA8 may be associated with schizophrenia in males.
\end{abstract}

S Afr J Psych 2013;19(3):86-87. DOI:10.7196/SAJP.455

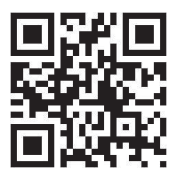

To the Editor. Numerous studies have shown that abnormal phospholipid metabolism may play a role in the aetiology of schizophrenia. Phospholipases A2 (PLA2) are a family of key enzymes in the metabolism of membrane phospholipids, which catalyse the hydrolysis of fatty acid at the sn-2 position of phospholipids. Increased PLA2 activity has been reported in the blood and brain tissue of schizophrenic patients, suggesting that disordered phospholipid metabolism plays a role in the disease. In addition, a genetic variant of a cytosolic PLA2 gene has been reported to be associated with schizophrenia. These data indicate that variants of PLA2 encoding genes are plausible candidates for increasing susceptibility to schizophrenia. A previous study showed a significant allelic and genotypic association between the calciumindependent phospholipases A2 (iPLA2) gene polymorphism and schizophrenia, ${ }^{[1]}$ suggesting that iPLA2 may play a role as a susceptibility gene for schizophrenia.

Patatin-like phospholipase domain containing 8 (PNPLA8) belongs to membrane-associated calcium-independent phospholipase A2. Interestingly, an earlier high-density genome scan indicated that a region of chromosome 7q31 where PNPLA8 is located likely harbours susceptibility genes for schizophrenia. ${ }^{[2]}$ However, to the best of our knowledge, the association between PNPLA8 and schizophrenia has not been investigated. Therefore, in the present study, we set out to determine whether there might be an association between 3 single nucleotide polymorphisms (SNPs) in PNPLA8 and schizophrenia in a Chinese population using a family trios sample.

A total of 201 Chinese parent-offspring trios of Han descent, consisting of fathers, mothers and affected offspring with schizophrenia, were recruited. These subjects originally came from the north-east area of China. The patients (154 males and 86 females), aged $25.9 \pm 6.7$ years, were admitted to a psychiatric hospital during the period between 2000 and 2009. All patients had a diagnosis of schizophrenia according to the International Statistical Classification of Diseases and Related Health Problems, 10th Revision (ICD-10) confirmed by two psychiatrists. The patients all gave signed, informed consent to participate in the study, which was approved by the ethics committee of the Jilin University.

Genomic DNA was extracted from whole blood samples using a genomic DNA extraction kit (Promega, US). The Liu et al.$^{[3]}$ method was used to genotype rs40876, rs10249427 and rs2396001 (Table 1).

The $\chi^{2}$ goodness-fit test showed that the genotypic distributions of 3 SNPs were not deviated from the Hardy-Weinberg equilibrium, in either the patient group or the parent group (both $p>0.05$ ). The transmission disequilibrium test (TDT) showed that rs40876 was the only SNP associated with schizophrenia in males $\left(\chi^{2}=4.667\right.$, $p=0.031)$. The other 2 SNPs did not show such an association (Table 1). In addition, there was no association between all 3 SNPs and schizophrenia in females. Analysis for haplotype transmission showed that the rs10249427 (C)-rs2396001(T) haplotype was associated with schizophrenic patients (adjusted $p=0.035$ after 10000 permutations), particularly among males (adjusted $p=0.024$ after 10000 permutations).

This study provides evidence linking variation in PNPLA8 with schizophrenia. The results in the present study suggest a small but significant contribution of a PNPLA8 polymorphism to susceptibility to schizophrenia in males in a section of the Chinese population of Han descent (north-east China). These findings are consistent with recent reports showing increased serum iPLA2 activity in 


\section{for

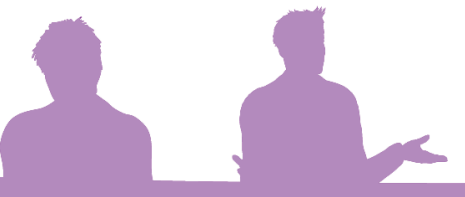

Table 1. The TDT for allelic association between SNPs and schizophrenia

\begin{tabular}{|c|c|c|c|c|c|c|c|c|c|}
\hline \multirow[b]{2}{*}{ SNPs } & \multicolumn{3}{|c|}{ Total } & \multicolumn{3}{|c|}{ Male } & \multicolumn{3}{|c|}{ Female } \\
\hline & Transmitted $^{\dagger}$ & $\chi^{2}$ & $p$-value & Transmitted $^{\dagger}$ & $\chi^{2}$ & $p$-value & Transmitted $^{\dagger}$ & $x^{2}$ & $p$-value \\
\hline \multirow[t]{3}{*}{ rs40876 } & & 1.607 & 0.205 & & 4.667 & $0.031^{\star}$ & & 2.579 & 0.108 \\
\hline & $\mathrm{G}=34$ & & & $\mathrm{G}=28$ & & & $\mathrm{G}=6$ & & \\
\hline & $\mathrm{T}=27$ & & & $\mathrm{~T}=14$ & & & $\mathrm{~T}=13$ & & \\
\hline \multirow[t]{2}{*}{ rs10249427 } & & 1.235 & 0.267 & & 1.719 & 0.190 & & 0.333 & 0.564 \\
\hline & $\mathrm{T}=86$ & & & $\mathrm{~T}=64$ & & & $\mathrm{~T}=22$ & & \\
\hline \multirow[t]{3}{*}{ rs2396001 } & & 0.962 & 0.327 & & 3.270 & 0.071 & & 2.400 & 0.121 \\
\hline & $C=99$ & & & $C=63$ & & & $C=36$ & & \\
\hline & $\mathrm{T}=109$ & & & $\mathrm{~T}=85$ & & & $\mathrm{~T}=24$ & & \\
\hline
\end{tabular}

first-episode patients with schizophrenia, ${ }^{[4]}$ suggesting perturbed lipid metabolism, especially with regard to iPLA2, in schizophrenia. However, whether the increased serum iPLA2 activity is associated with PNPLA8 polymorphisms deserves further investigation.

It is worth mentioning that the PNPLA8 rs40876 variant appears to contribute to schizophrenia only in males. There are two possible reasons for this. First, this might be due to the small number of female patients in our study. Second, this result suggests a role for sex hormones which may affect iPLA2 function and which could therefore be implicated in the aetiology of schizophrenia. Interestingly, a recent study showed that the BanI polymorphism in PLA2G4A (cytosolic PLA2; cPLA2) affects the mean age of schizophrenia onset only in male patients. ${ }^{[5]}$ Evidence suggests that sex differences among patients with schizophrenia have included age of onset, symptom severity, treatment response, course of illness, and outcome, favouring females, ${ }^{[6]}$ which may be associated with the neuroprotective action of female sex hormones in the brain. Therefore, the possible neuroprotective effects of female sex hormones in the presence of the PNPLA8 rs40876 could be checked in the context of schizophrenia. However, this is only our speculation; the sex difference in the association of the PNPLA8 rs40876 variant with schizophrenia warrants further investigation.
In summary, our present study has revealed a weak association between the PNPLA8 rs40876 polymorphism and schizophrenia in males, providing support for the membrane phospholipid hypothesis for schizophrenia. However, this finding remains preliminary and requires replication in other independent samples. Moreover, further studies with larger samples, especially in females, are required to better understand the influence of PNPLA8 polymorphisms in the regulation on the peripheral or brain iPLA2 activity in schizophrenia.

\section{References}

1. Junqueira R, Cordeiro Q, Meira-Lima I, Gattaz WF, Vallada H. Allelic association analysis of phospholipase A2 genes with schizophrenia. Psychiatr Genet 2004;14(3):157-160.

2. Detera-Wadleigh SD, Badner JA, Berrettini WH, et al. A high-density genome scan detects evidence for a bipolar-disorder susceptibility locus on 13q32 and other potential loci on 1q32 and 18p11.2. Proc Natl Acad Sci USA 1999;96(10):5604-5609.

3. Liu W, Tang Q, Jiang H, et al. Promoter polymorphism of interleukin-18 in angiographically proven coronary artery disease. Angiology 2009; 60(2):180-185.

4. Smesny S, Kinder D, Willhardt I, et al. Increased calcium-independent phospholipase A2 activity in first but not in multiepisode chronic schizophrenia. Biol Psychiatry 2005;57(4):399405

5. Nadalin S, Rubesa G, Giacometti J, et al. BanI polymorphism of cytosolic phospholipase A2 gene is associated with age at onset in male patients with schizophrenia and schizoaffective disorder. Prostaglandins Leukot Essent Fatty Acids 2008;78(6):351-360. [http://dx.doi. org/10.1016/j.plefa.2008.04.006]

6. Grossman LS, Harrow M, Rosen C, Faull R, Strauss GP. Sex differences in schizophrenia and other psychotic disorders: A 20-year longitudinal study of psychosis and recovery. Compr Psychiatry 2008;49(6):523-529. [http://dx.doi.org/10.1016/j.comppsych.2008.03.004] 